\title{
Explainable Deep Learning for Fault Prognostics in Complex Systems: A Particle Accelerator Use-Case*
}

\author{
Lukas Felsberger ${ }^{1,3}$, Andrea Apollonio ${ }^{3}$, Thomas Cartier-Michaud ${ }^{3}$, Andreas \\ Müller $^{2}$, Benjamin Todd ${ }^{3}$, and Dieter Kranzlmüller ${ }^{1}$ \\ 1 MNM-Team, Ludwig-Maximilians-Universität Muenchen, Oettingenstr. 67, \\ D-80538 München, Germany \\ 2 Hochschule Darmstadt, Haardtring 100, 64295 Darmstadt, Germany \\ 3 CERN, Route de Meyrin, 1211 Genève, Switzerland
}

\begin{abstract}
Sophisticated infrastructures often exhibit misbehaviour and failures resulting from complex interactions of their constituent subsystems. Such infrastructures use alarms, event and fault information, which is recorded to help diagnose and repair failure conditions by operations experts. This data can be analysed using explainable artificial intelligence to attempt to reveal precursors and eventual root causes. The proposed method is first applied to synthetic data in order to prove functionality. With synthetic data the framework makes extremely precise predictions and root causes can be identified correctly. Subsequently, the method is applied to real data from a complex particle accelerator system. In the real data setting, deep learning models produce accurate predictive models from less than ten error examples when precursors are captured. The approach described herein is a potentially valuable tool for operations experts to identify precursors in complex infrastructures.
\end{abstract}

Keywords: Prognostics and Diagnostics · Explainable AI · Deep Learning - Multivariate Time Series.

\section{Introduction}

Technical infrastructures are becoming increasingly complex while demands on availability are constantly rising. Failures of simple systems can often be manually analyzed in a relatively straight-forward manner by experts, whereas systems with increasing complexity and dependencies between infrastructures render manual failure analysis largely infeasible. This is primarily due to the sheer amount of potentially relevant failure precursors that must be considered.

In this study, modern particle accelerators are taken as example of complex infrastructures. Complete analytical models of failures and abnormal behaviors of particle accelerators are usually impossible; accelerators have numerous inter-operating subsystems, recording large amounts of diverse data, which

\footnotetext{
* This work has been sponsored by the German Federal Ministry of Education and Research (grant no. 05E12CHA). The final publication is available at Springer via http://dx.doi.org/10.1007/978-3-030-57321-8_8
} 
is difficult to analyse. In addition, the operational modes of particle accelerators can change over time, influencing the reliability and operating margins of sub-systems (e.g. an accelerator may operate as both an ion mode or a proton mode, with significant operating margins in one mode, and limited margins in the other). Moreover, an accelerator is a continuously evolving infrastructure, maintenance is carried out, sub-systems are upgraded and evolved, modes of operation are tuned, and adjusted. All combined, this makes traditional modelling approaches inadequate.

In general, operation data, such as system alarms, events, faults, physical measurements, etc., are abundant and are logged at rates and dimensionalities which are impossible to analyze in real time by a human operator. Hence, automated data driven analyses are required to assist human operators in diagnosis of system operation.

For application in particle accelerator environments, data driven methods need to handle heterogeneous data sources (e.g. binary alarms, discrete logged settings, continuous monitoring values), function with raw data and extract features automatically, learn from few observed failures and many observed nonfailures (imbalanced data), and scale to several hundreds of input signals.

A data driven prognostics and diagnostics framework should reveal both (a) prediction of failures and alarms in advance and (b) clear insights for the interpretation of failure predictions. This would allow operators to increase infrastructure availability by preventive and pro-active actions to mitigate or remove failure conditions.

Related work Methods to predict faults have been reviewed extensively in the fields of prognostics and diagnostics $[13,31,25]$, system health management [15] and predictive maintenance [23]. They are commonly classified as model driven, when a-priori modeling of the system behaviour is employed, or data driven, when the system behaviour is inferred from data. As model driven approaches are becoming infeasible when prior knowledge on the infrastructure behaviour is limited, only data driven methods are considered here.

Within data driven approaches, a distinction can be made between classical Machine Learning (ML) (e.g. support vector machine, k-nearest neighbour, decision tree), deep learning (e.g. deep belief networks, convolutional neural networks, recurrent neural networks) and probabilistic reasoning (e.g. Hidden Markov models, Gaussian Processes, Bayesian Graphical Networks) methods. Traditional ML is computationally efficient but often requires extensive data pre-processing and feature extraction efforts. Deep learning allows phenomena to be modelled in greater detail with reduced preprocessing, when sufficient data is available. Probabilistic reasoning allows to quantify and propagate uncertainty when limited data is available, but cannot match the computational effectiveness of traditional ML.

Further possible classifications are based on the field of application (e.g. mechanical systems, electronics, software), the complexity of the studied system (e.g. component, unit, system, system of system, human interaction), the type of learning (supervised, semi-supervised, unsupervised) and the kind of data used 
(univariate, multivariate, binary, numeric, discrete, text, raw data, features). Methods are usually not classified by the interpretability and explainability of their predictions, which is an important criterion for the considered use case and for many other applications [1].

Support-Vector Machines (SVMs) play a large role within traditional ML approaches. Zhu et al [32] and Fulp et al [12] developed SVM based methods to predict the failures of hard drives based on features indicating system health. Leahy et al [16] predicted wind turbine failures based on features of data from Supervisory Control and Data Acquisition (SCADA) systems in wind turbines. Fronza et al [11] extended the approach to systems of systems by predicting failures in large software systems. SVMs would allow interpretation of trained models, especially with linear Kernel functions. However, none of the authors considered investigating the structure of the learned models in order to gain insights into the failure mechanisms of the systems.

L1 regularized Granger causality was developed by Qiu et al [22] to detect root causes of anomalies in industrial processes. They reported interpretable results, scalability and robust performance. However, the method was not used to predict faults.

Association rule mining based methods were used by Vilalta et al [28] and Serio et al [27] to identify failure mechanisms in complex infrastructures using interpretable models. Vilalta et al detected anomalies in computer networks. The class imbalance problem is overcome by learning only from the minority class representing anomalies. They reported good accuracy but limited applicability of the method. The work by Serio et al represents the only relevant application in the particle accelerator domain. The authors successfully extracted expert verified fault dependencies between subsystems from logging data. However, time dependence between events were not considered and fault predictions are therefore not possible.

As an example of probabilistic reasoning methods, Mori et al [19] proposed a Bayesian graphical model approach to perform root cause diagnosis in industrial processes. The method is interpretable and accurate. At the overlap of probabilistic modeling and deep learning is the work of Liu et al [17]. The framework combines ideas from state space modeling with Restricted Boltzmann Machines or Deep Neural Networks to identify root causes of anomalies in industrial processes. High accuracy and scalability were reported.

With deep learning methods Saeki et al [24] classified wind turbine generator anomalies from spectral data. On a test data set, a visual explanation technique attributed importance to the same failure precursors as human experts. They noted that the data set used was not representative for real world scenarios. Amarsinghe et al [2] detected Denial of Service attacks in computer networks using a Deep Neural Network and a so called Layer-wise Relevance Propagation (LRP) introduced by Bach et al [4] to highlight relevant inputs for classification decisions. High classification accuracy was reported and explanations were intuitive to interpret. However, the method used features generated from raw data. Bach-Andersen et al [5] performed an extensive study of early fault precursor 
detection for ball bearings in wind turbines using raw spectral data. They compared logistic regression, fully connected neural networks and deep convolutional neural networks across three classification tasks. The deep network was found to perform the best. Using a visualization technique of higher layers of the trained deep network, the strong performance of the network could be explained and insights about the failure behaviour were derived. The method yielded accurate results, handled class imbalance and scaled well.

Demonstrating performance advantage and universality, explainable deep learning frameworks seem promising for fault prediction in the accelerator domain. The work of Bach-Andersen et al provides a strong baseline but it was optimized for a different application domain and used a data structure which is not compatible with the particle accelerator use case. Moreover, the LRP mechanism by Bach et al was more intuitive to the authors of this study than the high level feature visualization used in Andersen et al. Furthermore, LRP is based on a more firmly established theory $[18,26]$. A recent extensive review by Fawaz et al [9] on deep learning architectures for time series problems reveals that convolutional neural network structures outperform traditional methods across a variety of multivariate time series classification tasks. Similar findings were previously reported by Wang et al [29] for univariate time series. Therefore, we chose neural network architectures as suggested in Fawaz et al for failure prediction and LRP by Bach et al as the explanation mechanism for the framework presented in this study. The goal is to evaluate whether such a framework can successfully be applied as fault prognostics and diagnostics tool in the accelerator domain and whether it provides significant advantages over classical ML methods. It is the first of its kind in the particle accelerator domain based on state-of-the-art deep learning for multivariate time series and explainable AI methods.

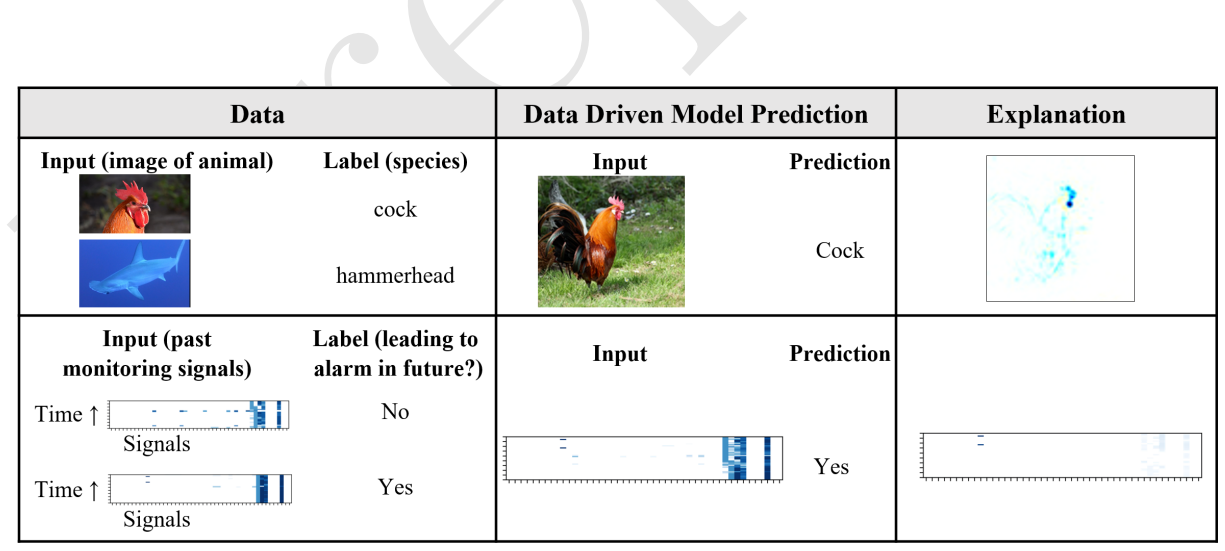

Fig. 1. Upper Row: Machine learning algorithms are able to identify animal species based on labeled images. Explanation techniques help to understand which pixels contribute the most to assign a certain species to an input image. [4] Lower Row: In the same way, a machine learning algorithm can learn a model to predict infrastructure failures based on time series data of monitoring signals. Here explanation techniques provide information about the most relevant signals leading to the failure. 
The idea of the proposed framework is illustrated in Fig. 1. Time series of data are obtained during the operation of complex infrastructures, such as particle accelerators. Operational alarms or anomalies lead to specific fault events in the data. A sliding window approach can extract snapshots of the machine behaviour before the occurrence of fault events and snapshots during normal operation. Thereby, a supervised training data set is generated without manual labeling effort. From such data, a discriminator, such as a deep neural network, can learn general rules to predict if a certain system snapshot will lead to failures after a certain time. If a fault is predicted, LRP highlights the most relevant fault precursors which help system experts understand the fault mechanism and take preventive measures.

The framework is introduced in Section 2. In Section 3 the effectiveness and the suitability of the framework are evaluated in experiments with synthetically generated time series data. Then, the method is applied to real world logging data sets of a particle accelerator to verify its suitability for the accelerator domain. Throughout, the deep learning frameworks are compared to classical machine learning methods, such as support-vector machines, random forests, and k-nearest-neighbor classifiers. Summary, Conclusions and Outlook are given in Section 4.

\section{Methodology}

Definitions and Overview The subject of study is an infrastructure $\mathbf{I}$ which can be composed of multiple sub-systems. A range of $N$ observable signals, monitors the behaviour of the infrastructure and its environment over time, forming a multivariate time series, $\mathbf{S}=\left\{\mathbf{S}_{i, t}: i \in[1: N]\right.$ and $\left.t \in \mathbb{N}\right\}$. These can include logged continuous and discrete parameters, event- and alarm-logs, inputand output-signals, etc. The infrastructure has a range of failure modes which indicate certain malfunctions. These failure modes are observable in a subset of signals.

An autoregressive model predicts the future behaviour of a system based on its current and past states. For a complex infrastructure, it can be expected that failures may appear without announcing themselves in advance by precursors. Even for situations with advance precursors, not all relevant processes might be monitored. Therefore, an autoregressive model can only approximate future failures based on time-discrete monitoring signals of complex infrastructures,

$$
\mathbf{S}_{N,\left[t+t_{p} \delta t: t+\left(t_{p}+n_{o}\right) \delta t\right]}^{F} \approx \mathbf{\Phi}\left(\mathbf{S}_{[1: N],\left[t-n_{i} \delta t: t\right]}^{P}\right)
$$

with

$-\mathbf{S}_{N,\left[t+t_{p} \delta t: t+\left(t_{p}+n_{o}\right) \delta t\right]}^{F}=1$, if a failure occurs between time $t+t_{p} \delta t$ and time $t+\left(t_{p}+n_{o}\right) \delta t$, and zero otherwise,

$-\mathbf{S}_{[1: N],\left[t-n_{i} \delta t: t\right]}^{P}$ being finite histories of observed signals covering the time stamps $t-n_{i} \delta t$ to $t$ and being considered as possible precursors,

- $\delta t$ being the discretization time, 
- $t_{p}$ the prediction- or lead-time,

- $n_{o}$ the number of time steps chosen to capture the future failure behaviour,

- $n_{i}$ the number of discrete time steps chosen to capture the history of the observed signals and

- $\boldsymbol{\Phi}$ an auto-regressive model,

as illustrated in Fig. 2.

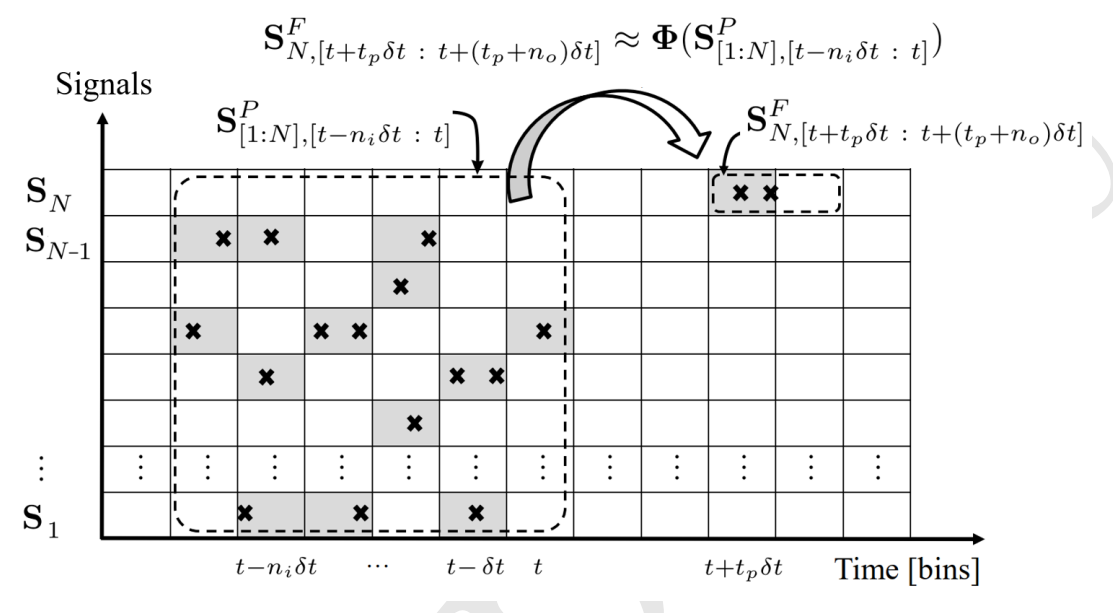

Fig. 2. Time discrete model formulation. The $\mathrm{x}$-axis represents discrete time and the y-axis monitoring signals of the investigated infrastructure. Crosses mark events which could be faults, alarms, changes in monitoring values, etc. Events of the signal $\mathbf{S}_{N}$ represent infrastructure faults that the model $\boldsymbol{\Phi}(\cdot)$ predicts.

In a few simple cases, the model $\boldsymbol{\Phi}(\cdot)$ can be obtained from first principles. In the case of complex infrastructure, the model needs to be determined in a data driven fashion using machine learning or time series analysis techniques. Models can learn the system behaviour from time series data in a supervised fashion by supplying pairs of input-data, $\mathbf{S}_{[1: N],\left[t-n_{i} \delta t: t\right]}^{P}$, and output- or target-data, $\mathbf{S}_{N,\left[t+t_{p} \delta t: t+\left(t_{p}+n_{o}\right) \delta t\right]}^{F}$, of the observed history of the studied infrastructure.

Once such a model has been trained, it can predict failures when supplied with observed data of the infrastructure. However, acting as black box it is not able to provide operators of the infrastructure any further information concerning the predicted failures, such information could be used for preventive actions to prevent the reoccurence of the failure observed.

To address this, the framework provides a relevance measure, $\rho\left(\mathbf{S}^{P}\right) \in \mathbb{R}^{\left(n_{i}, N\right)}$, of each of the observed signals at each time step, which signifies the most relevant input signals for model predictions. This can be provided to infrastructure operators, to focus their attention on a range of potential precursors. The framework does not search for causality but temporal precedence of correlated failure 
precursors [8]. Including expert verified causal dependencies, it may be possible to generate model based descriptions of failure behaviour [10].

The framework can be used as a real-time analysis tool, acquiring data continuously from the infrastructure as an input, predict imminent failures as an output. In this manner it could advise operators to take preventive measures. These actions require a certain lead-time, $t_{p}>0$, to allow the system operators time to intervene, $t_{p} \delta t$, to preemptively act on predicted failures. As a postmortem tool, the framework could be used without lead-time constraints, in the analysis and explanation of complex failure mechanisms, which could then be investigated by system experts to mitigate reoccurrence. All of these considerations are particularly relevant for future generations of high-energy particle accelerators, for which the increasing size (order of $100 \mathrm{~km}$ ), are expected to set unprecedented challenges in terms of maintainability of the infrastructures.

Machine Learning Pipeline This Subsection describes in more detail how predictive models $\boldsymbol{\Phi}(\cdot)$ are learned from observed monitoring data.

Data Collection The observable signals $\mathbf{S}$ of the studied infrastructure are stored in time series format in a data-set $\mathcal{D}$. Based on a-priori expert knowledge, a preselection of potentially relevant signals can be chosen to reduce the required storage capacities. Further details on the data collection will be given for the use cases 3 .

Model Selection and Evaluation The problem formulation contains a range of hyperparameters to be optimized, e.g. $\left[\delta T, n_{i}, n_{o}, t_{p}\right]$, which are introduced later in this Section. To do so, a K-fold validation strategy is adopted for the studied use-cases [6]. It is performed by splitting the overall data-set, $\mathcal{D}$, in a training set, $\mathcal{D}_{\text {train }}$ up to time $t_{\text {split }}$, and a final test set, $\mathcal{D}_{\text {test }}$ after time $t_{\text {split }}$. The K-folds for hyperparameter selection are obtained by a further splitting of the training set into $K$ sub-training sets at splitting times $t_{s u b-s p l i t, k}, k=1, \ldots, K$.

Subsampling As failures in the considered infrastructures are rare, the target data $\mathbf{S}_{N}^{F}$ will contain few failures and therefore contain many more '0's (no failures) than '1's (failures). Such an imbalanced data-set requires subsampling of the majority class (usually class ' 0 ') randomly until a certain target ratio $p_{0, t a r g}=f r e q\left(c l_{0}\right) / f r e q\left(c l_{1}\right)$, is reached. Here, freq $\left(c l_{x}\right)$ denotes the number of class 'x' instances in the data set. After subsampling, data $n_{\text {cov }}$ time-steps before and after each class ' 1 ' instances are added to the training set as it leads to better performance of the learned models. This can be seen as a method to increase 'contrast' in the class ' 1 ' neighbourhood. 
Input Filtering and Normalization Input signals having values equal to zero or less than $\alpha_{\min }{ }^{4}$ times or having a variance smaller or equal to $\sigma_{\min }{ }^{5}$ are automatically removed. After the filtering, the input signals of the training data are normalized to the range $[0,1]$.

Model Learning Algorithms To learn a model $\mathbf{\Phi}(\cdot)$, from pairs of historical inputand output-data of the infrastructure, deep learning algorithms and classical machine learning algorithms were used. The target variables take values ' 0 ' and '1', so the failure forecasting problem has been formulated as a binary classification task.

Based on recent studies on using deep learning for multivariate time series classification, the study is focused on fully-convolutional neural networks. They reach state-of-the-art performance while being faster to train than recurrent neural networks $[9,29]$. These deep networks were compared against SVM, Random-Forest, and K-Nearest-Neighbour classifiers, representing classical ML techniques. The following algorithms were used:

- FCN: The classifier is based on an architecture proposed by Wang et al [29]. It consists of three convolutional blocks with three operations in each: a convolution is followed by a batch normalization [14] and fed into a ReLU activation. The output of the last convolutional block is averaged over the whole time-dimension in a Global Average Pooling layer (GAP). Lastly, the GAP's output is fully connected to a traditional softmax classifier.

The convolutions are characterised by having a stride of 1 with zero padding to conserve the shape of the input data. The three convolution layers contain 128,256 , and 128 filters with a filter length of 8,5 , and 3 , respectively. It was selected as it achieved the highest accuracy across 13 multivariate time series datasets in the review by Fawaz et al [9]. We use the implementation of the review paper with minor modifications. The number of training epochs is set to 2000. An early stop criterion ensures that training is terminated if the validation loss is not decreasing by more than 0.001 after 200 epochs, with the loss function set to categorical cross-entropy.

- FCN2drop: This is the same classifier as FCN, except with dropout applied to two layers in the network. Dropout is applied on the second convolution and on the GAP layer with a dropout probability of $p_{\text {drop }}=0.5$. Due to the scarcity of class '1' items in the learning data, dropout regularization is expected to prevent overfitting of the network.

- FCN3drop: This is the same classifier as FCN, except with dropout applied to three layers in the network. Dropout is applied on the second and third convolution and on the GAP layer with $p_{\text {drop }}=0.7$.

- tCNN: As proposed by Zhao et al [30], the network consists of two convolutional layers with 6 and 12 filters, respectively. The final layer is a traditional fully-connected layer with sigmoid activation function. It uses the

\footnotetext{
${ }^{4}$ Unless stated otherwise, $\alpha_{\min }=4$, as it was the minimal number of examples from which the selected algorithms could learn from. [3]

${ }^{5}$ Unless stated otherwise, $\sigma_{\min }=0$, to only remove constant signals which do not contain any discriminatory information.
} 
mean-squared error instead of cross-entropy as loss function. We have taken the implementation from Fawaz et al using the same early stopping criterion as for the FCN models.

- SVM: A support vector machine with linear kernel functions, using the implementation from [21] with default parameters.

- RF: A random forest classifier is a meta classifier based on decision trees. We used the implementation from [21] with the number of features to consider for optimal splitting set to the square-root of the number of features and default parameters.

- kNN: A k-Nearest-Neighbour classifier based on the implementation from [21] using $n=7$ neighbours and default parameters.

The classifier parameters were manually pre-selected based on recommendations in the Scikit-learn user guide [21] and preliminary experiments on data-sets as described in Section 3. SVM, RF and kNN classifiers require the input to be onedimensional. Therefore, the $2 \mathrm{D}$ multivariate time series input data is flattened to one dimension when fed into the classifier for training and prediction.

To evaluate the performance of the classifiers the accuracy and the F1 score on the test-set is examined. Due to the imbalance of classes, additionally the fraction of the majority class in the test-set is reported.

Explaining Predictions In order to help operation experts interpreting predictions of the framework and discovering failure mechanisms, the relevance of each input at discrete time steps in the observed history, $\rho\left(\mathbf{S}^{P}\right) \in \mathbb{R}^{\left(n_{i}, N\right)}$, is quantified and reported.

For deep learning methods, several such relevance reporting techniques have been developed. The so-called LRP was chosen for implementation as it provides best-in-class explanations [26]. It is based on a backward pass within the neural network which is layer-wise relevance conserving. Neurons contributing the most to the following layer receive most relevance from it during its backwards pass.

Testing the Gradient $x$ Input [18], LRP-0, and LRP- $\epsilon$ rules [4], the LRP-0 rule showed a better omission of irrelevant failure precursors with synthetic test data.

For the classical machine learning methods, the input relevance for the SVM classifier was calculated by evaluating the input feature weight vector [21]. The input relevance is depicted as $2 \mathrm{D}$ heatmaps with more relevant inputs being represented in darker colours.

\section{$3 \quad$ Numerical Experiments}

The method described above is applied to several use-cases, which are briefly introduced. Implementations are available on github ${ }^{6}$.

\footnotetext{
${ }^{6}$ https://github.com/lfelsber/alarmsMining
} 
Synthetic Data Experiments The framework is tested with synthetically generated data. It resembles characteristics of real-world data-sets but with the advantage of a known ground truth which allows to verify if the correct failure precursors are identified.

Noise Robustness In this experiment, an infrastructure is modeled by $n_{\text {rand }}$ systems firing precursors randomly and one system firing two consequent failure precursors which are always followed by an infrastructure failure $S_{s}^{F}$. The timing of the alarms is illustrated in Fig. 3. The goal is to study the ability of the framework to filter and explain the deterministic pattern at increasing numbers $n_{\text {rand }}$ of randomly firing systems despite being provided only less than ten failures to learn from.

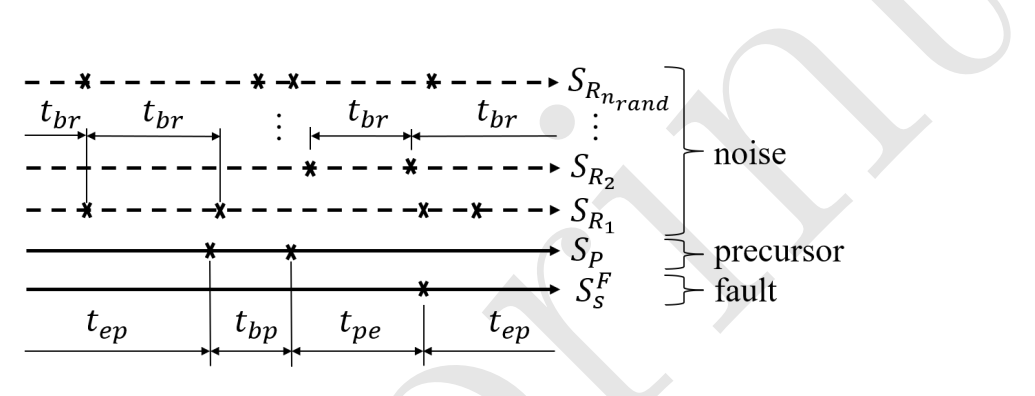

Fig. 3. Parameters of synthetic pattern.

The synthetic data is generated with a time $t_{b r} \sim \mathcal{N}(\mu=14.61 d, \sigma=$ $14.61 d)$ between randomly firing precursors, $S_{R_{l}}, l=1,2, \ldots, n_{\text {rand }}$, a time $t_{b p} \sim \mathcal{N}(\mu=1 d, \sigma=1 d / 24)$ between deterministic precursors $S_{p}$, a time $t_{p e} \sim \mathcal{N}(\mu=10 d / 24, \sigma=1 d / 24)$ between deterministic precursors $S_{p}$ and infrastructure failures $S_{s}^{F}$, and a time $t_{e p} \sim \mathcal{N}(\mu=36.525 d, \sigma=36.525 d)$ between infrastructure failure $S_{s}^{F}$ and deterministic precursors $S_{p}$ with $d$ being a day of 24 hours. The data is generated for a time range of 2.7 years and $n_{\text {rand }}$ being $[1,2,4,8,16,32,64,128,256,512]$.

The framework is applied with sampling times $\delta t=[2 h, 3 h]$ ( $h$ for hours), an input range $n_{i}=40$, a lead-time $t_{p}=0$, an output range of $n_{o}=[1,2,3,4]$, a sub-sampling target ratio $p_{0, \text { targ }}=0.8$, and a class ' 1 ' neighbourhood coverage $n_{\text {cov }}=2$. We split the data set with a splitting time $t_{\text {split }}$ chosen so that 80 percent of the data-set are used for training and model-selection and 20 percent for final testing. Training and model selection is performed by a 7 -fold validation for sub-splitting times $t_{s u b-s p l i t}$ chosen so that [50, $\left.55,60,65,70,75,80\right]$ percent of the training data set are used for training and $[50,45,40,35,30,25,20]$ percent for validation. On average 7 (13) infrastructure failures were in the training data of the validation folds (the whole data-set). Hence, we are investigating a small data scenario.

Of the 4480 trained models, we present the results for $\delta t=3 h$ and $n_{o}=2$ as these parameters led to good results for all classifiers. In Fig. $4 \mathrm{a}$ and $4 \mathrm{~b}$, the 


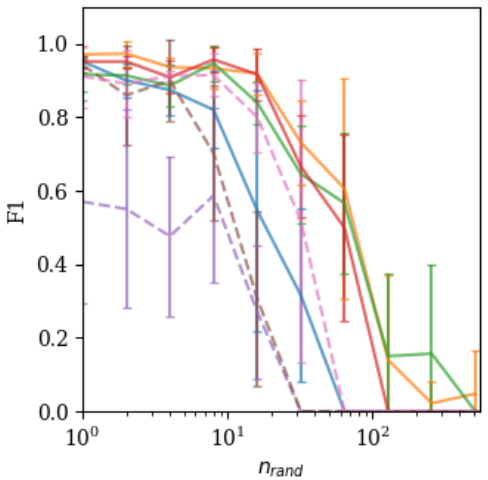

(a)

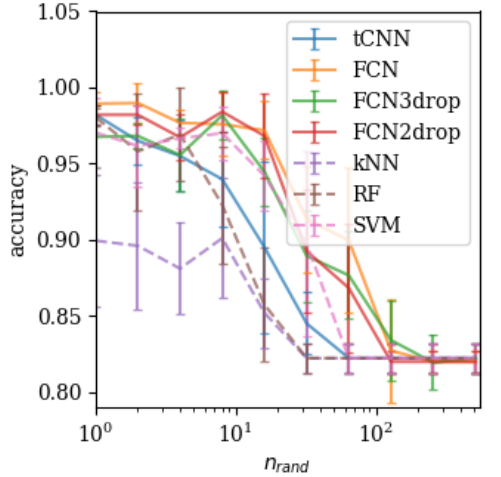

(b)

Fig. 4. Dependency of the predictive performance on the number of randomly firing signals. The line depicts the mean and the error bar plus and minus one standard deviation calculated over the 7 validation sets. Solid lines represent deep models which perform on average better than the classical models (dashed). (a) The F1 score. Note that the predictors level out at 0.0 for large $n_{\text {rand }}$, which is the binary F1 score when always predicting the majority class (' 0 '). (b) The accuracy. Note that the predictors level out at 0.82 for large $n_{\text {rand }}$, which is the accuracy when always predicting the majority class ('0').

performance metrics are plotted as a function of the number of randomly firing signals, $n_{\text {rand }}$. Evidently, for higher numbers of random signals the performance is decreasing. Nevertheless, the correct patterns can be identified out of up to 100 random signals from as little as 7 examples in the training sets with the chosen parametrization. This implies that in a real-data scenario, less than 10 training examples can be sufficient to detect failure patterns.

Further characteristics of the framework have been studied in [3]. It has been found that increasing $n_{o}$ by one or two can lead to accuracy improvements especially when the duration between precursors and failures has a high variance. Other insights are that the input relevance highlights the precursors with the lowest timing variance, and that patterns can be found from as few as four failure examples.

Recovering Fault Tree Structure Often faults in infrastructures are due to the interaction of multiple sub-systems. This experiment tests if the framework is able to identify failures due to interaction of sub-systems and if it can be explained by a system operator.

To do so, synthetic data is generated by simulating multiple sub-system interactions leading to infrastructure failures as illustrated in Fig. 5. An infrastructure failure, $S_{b}^{F}$, occurs after two precursor signals, $S_{P_{1}}$ and $S_{P_{2}}$, fulfill either a Boolean AND, OR, or XOR condition. Four additional noise signals, $S_{R_{1-4}}$, 
are added to simulate non-interacting parts of the infrastructure. Time delays between signals are chosen to represent a realistic scenarios.

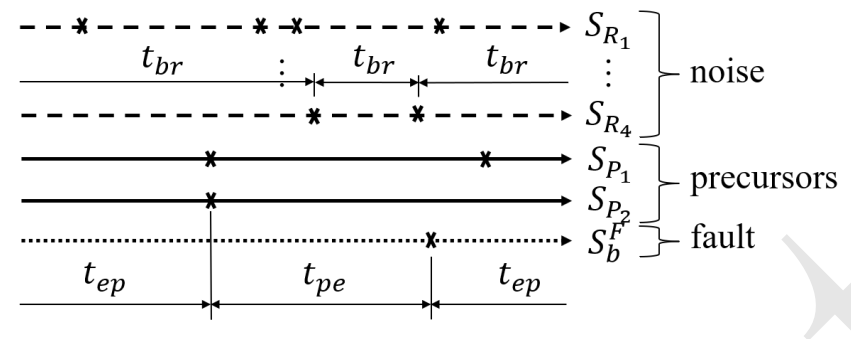

Fig. 5. Parameters of synthetic pattern.

The results for data generation parameters $t_{b r} \sim \mathcal{N}(\mu=23 \mathrm{~min}, \sigma=24 \mathrm{~min})$, $t_{p e}=71 \mathrm{~min}, t_{e p}=120 \mathrm{~min}$ are shown in Fig. 6 . The framework is applied with sampling time $\delta t=12 \min$ ( $\min$ for minutes), an input range $n_{i}=5$, a lead-time $t_{p}=5$, an output range of $n_{o}=1$, a sub-sampling target ratio $p_{0, t a r g}=0.8$, and a class '1' neighbourhood coverage $n_{\text {cov }}=2$. The data set was split in an equally sized training and testing set without additional K-fold validation as no hyperparameter selection was performed. The input data contain less than 20 examples of infrastructure failures. Deep networks and traditional ML algorithms perform well, consistently reaching $F 1>0.97$. The results for the FCN2drop network are detailed below.

Fig. 6 shows three randomly selected input windows from the test data set with subsequent infrastructure failures for the AND, OR, and XOR scenario. Left columns show the unfiltered input window and right columns show the explanations with relevant signals as obtained from the FCN2drop network. Clearly, the correct precursors, $S_{P_{1}}$ and $S_{P_{2}}$, could be identified by the framework and the noise, $S_{R_{1}-4}$, filtered. Comparing the groups of three input windows, the Boolean logic can be reconstructed. However, this relies on comparing different situations and is not possible from a single image. Still, the results confirm that the framework can identify failures due to interaction of multiple subsystems and allows system operators to explain the interactions. The inputs (left columns) would not expose the fault logic without the filtering by the explanation framework (right columns).

Particle Accelerator Data Experiments Logging data from a particle accelerator infrastructure operated at CERN is used. The so-called Proton Synchrotron Booster (PSB) has a radius of $25 \mathrm{~m}$ and is composed of four superimposed rings. It continuously logs failure modes, alarm data, operational settings data, physical monitoring and condition data, among others. The goal is to learn predictive models of failure modes and their mechanisms from data stored between January 2015 and December 2017. The learning task is difficult as the 


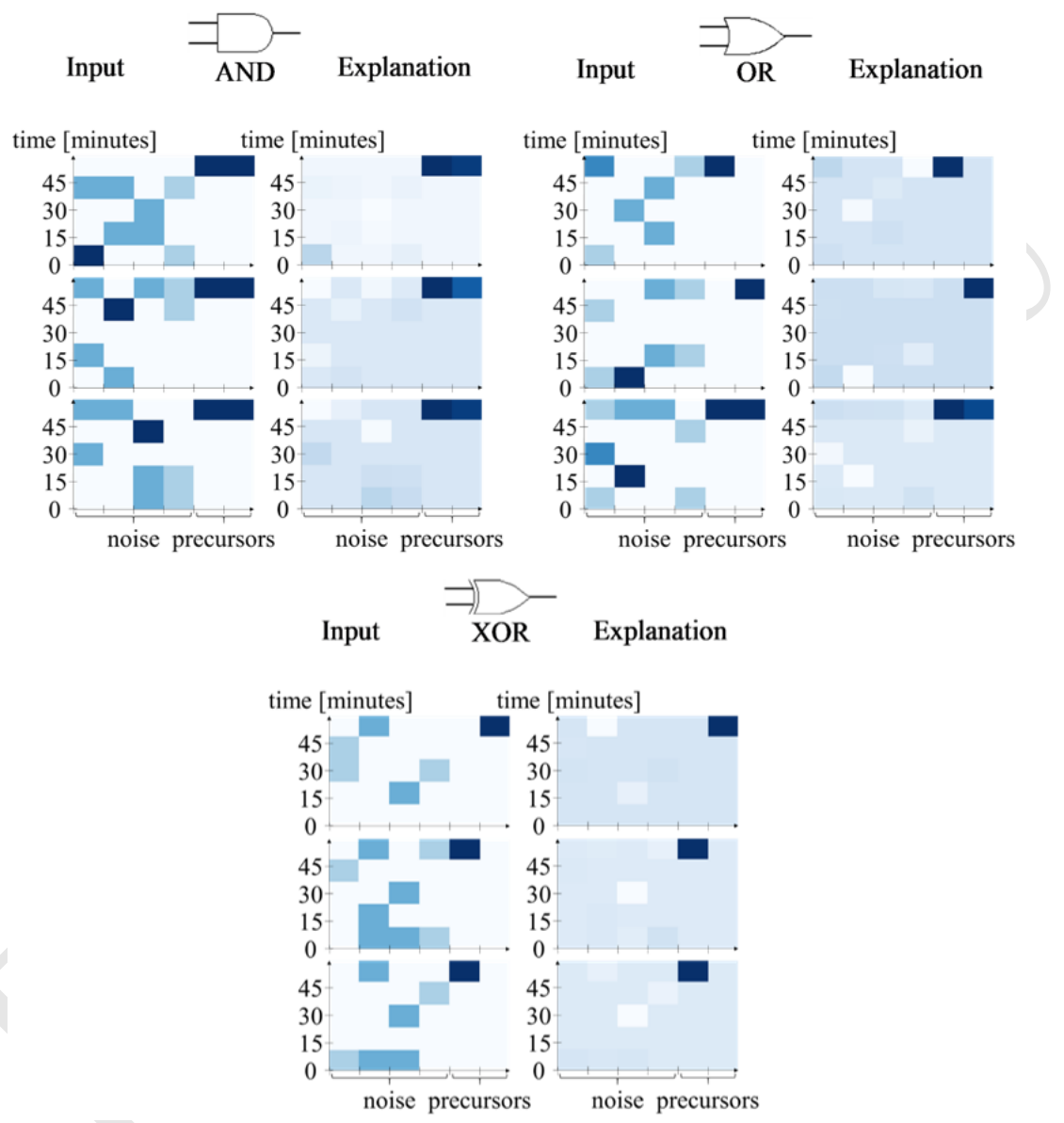

Fig. 6. Illustration of AND, OR and XOR fault logic extraction. Left columns show three randomly selected input windows before failure occurrence. Right columns show the relevant precursors obtained with the FCN2drop network (darker colours indicate higher relevance). Comparing the relevant precursors (right columns), allows to distinguish different Boolean rules and recover the fault logic of the system. 
infrastructure is continuously worked on and modified, the data logging mechanisms have not been designed for historical data analysis, and failure data are rare [20]. As for any real-world infrastructure, only a subset of all relevant processes leading to failures can be observed. Furthermore, only system operators and experts can verify if the framework highlights the correct relevant precursors.

The goal is to forecast failures of eight power converters used in the PSB. The following signals are used for the analysis:

- Alarm logging data from the LASER alarm system [7]. The alarms are characterised by a system name, fault code and priority. The priority can be either 2 or 3 for the selected data. Priority 2 leads to a warning, whereas, priority 3 leads to a shutdown of the system. We select the ten most frequent priority 3 fault types of the 8 investigated power converters as the failure signals $\mathbf{S}_{O, F}$ we attempt to predict. The alarms are logged with a rising and falling flag indicating the beginning and the end of the alarm state, respectively. Only the rising flag is used as data. Data is grouped by system name for the input. This leads to eight input signals.

- Interlock signals register external and internal disturbances which potentially lead to the shut-down of the infrastructure. We have selected 27 signals based on operations experts' recommendations.

- The beam destination variable is an indicator of the operational mode of the PSB. The eight different beam destinations are hot encoded and added to the input data.

Time periods in which the PSB is switched off for maintenance were removed, as the alarm data is not valid during these periods of time.

Mixing Synthetic and Real Data In a first attempt, the goal is to test if a known pattern can be extracted from the real-world data. Therefore, the noise robustness experiment was repeated with the randomly firing systems being replaced by real-world data containing 8 LASER alarm signals, 27 interlock signals, and 8 beam destination signals from the PSB.

The framework was applied to the data with sampling times $\delta t=[2 h, 3 h]$ ( $h$ for hours), an input range $n_{i}=40$, a lead-time $t_{p}=0$, an output range of $n_{o}=[1,2,3,4]$, a sub-sampling target ratio $p_{0, \operatorname{targ}}=0.8$, and a class ' 1 ' neighbourhood coverage $n_{\text {cov }}=2$. The same model validation strategy is chosen as for the synthetic data experiment. All classifiers were trained and evaluated.

Of the 448 trained models, the results for $\delta t=3 h$ and $n_{o}=3$ achieved high F1 and accuracy and are presented in Table 1 . The FCN networks show a strong performance in this experiment reaching $\mathrm{F} 1$ close to 1 based on only 7 training examples. This indicates that patterns in the real-world data can be detected from less than ten examples. Fig. 7a shows the input activation for the FCN and SVM, respectively. Both correctly identify the relevant precursor out of 43 signals.

Real Data The framework is tested to determine whether it can predict and explain the ten most frequent priority 3 failures in the PSB accelerator power 


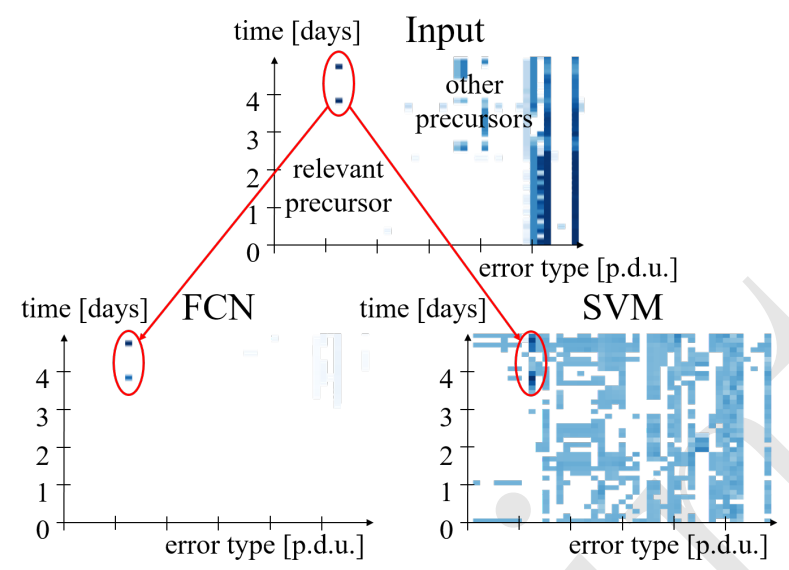

(a)

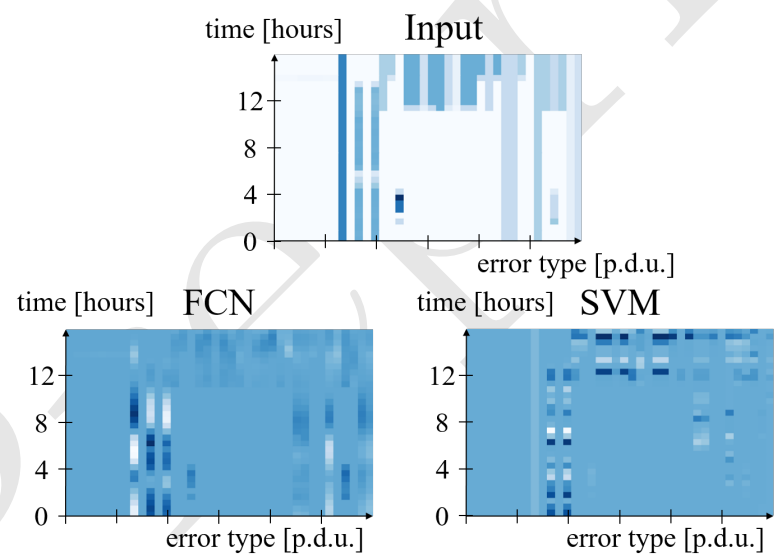

(b)

Fig. 7. (a) Upper: Input data for a single example of class ' 1 ' in the test set (not shown, occuring shortly after day 5). Lower left: Correctly identified relevant failure precursors by FCN network. Lower right: Correctly identified failure precursors by SVM network across all class '1' examples in the test set. Note that FCN and SVM evaluate non-relevant error messages differently in their models (darker colours in the heatmap signify higher relevance). (b) Input relevance for real data snapshot with $\delta t=30 \mathrm{~min}$, $n_{i}=32, t_{p}=0, n_{o}=4$ and $\mathbf{S}_{F_{0}}$. The relevance in the upper region is higher for SVM. System experts could identify that certain combinations of external interlock signals and operational modes leading to infrastructure failures. 
Table 1. Performance metrics for mixing synthetic and real data experiments. frac maj $_{\mathbf{m}}$ stands for the fraction of the majority class and is shown as reference for the accuracy of a trivial predictor always predicting the majority class. $v$ and $\sigma_{\mathbf{v}}$ stand for the mean and standard deviation over the 7 validation folds, respectively, and $t$ for results on the test set.

\begin{tabular}{|c|c|c|c|c|c|c|c|c|c|c|c|c|c|c|c|c|c|c|c|c|}
\hline & FCN & & $\mathrm{CN} 3$ & 3dro & op FC & $\mathrm{CN} 2$ & 2 dro & & & NN & & $\mathbf{k N}$ & & & RF & & & SVM & & frac maj \\
\hline & $\begin{array}{lll}v & \sigma_{v} & t\end{array}$ & & $v$ & $F_{p}$ & $t$ & $v$ & $\sigma_{v}$ & $t$ & & $\sigma_{v}$ & & $\sigma_{n}$ & $t$ & $\underline{v}$ & $\sigma_{v}$ & $t$ & $\underline{v}$ & $\sigma_{v}$ & $t$ & $\begin{array}{lll}v & \sigma_{v} & t \\
\end{array}$ \\
\hline $\mid 0.9$ & $\begin{array}{l}.970 .030 .9 \\
890110.9\end{array}$ & & .930 .0 & $\begin{array}{ll}06 & 0.5 \\
22 & 0.8\end{array}$ & $\begin{array}{l}.950 .9 \\
800.9\end{array}$ & 970. & $\begin{array}{l}.020 .9 \\
0509\end{array}$ & & 830 & .030. & & & & & $\begin{array}{l}330.03 \\
0.00\end{array}$ & & & $\begin{array}{l}910.05 \\
730.15\end{array}$ & .9 & \\
\hline
\end{tabular}

converters using the data introduced above. All signals are chosen as input data and the ten most active failure signals within the data set $\left(\left[\mathbf{S}_{F_{0}}, \ldots, \mathbf{S}_{F_{9}}\right]\right)$ were predicted.

The framework is applied with sampling times $\delta t=[10 \mathrm{~min}, 30 \mathrm{~min}, 2 h](h$ for hours, $\min$ for minutes), input ranges $n_{i}=[16,32,64]$, lead-times $t_{p}=[0,1]$, output ranges of $n_{o}=[1,2,4,16]$, a sub-sampling target ratio $p_{0, t a r g}=0.8$, and a class ' 1 ' neighbourhood coverage $n_{\text {cov }}=2$. The same model validation strategy is chosen as for the synthetic data experiment.

Table 2. Performance metrics for real data experiments. frac $\mathbf{m a j}_{\mathbf{m}}$ stands for the fraction of the majority class and is shown as reference for the accuracy of a trivial predictor always predicting the majority class. $v$ and $\sigma_{\mathbf{v}}$ stand for the mean and standard deviation over the 7 validation folds, respectively, and $t$ for results on the test set.

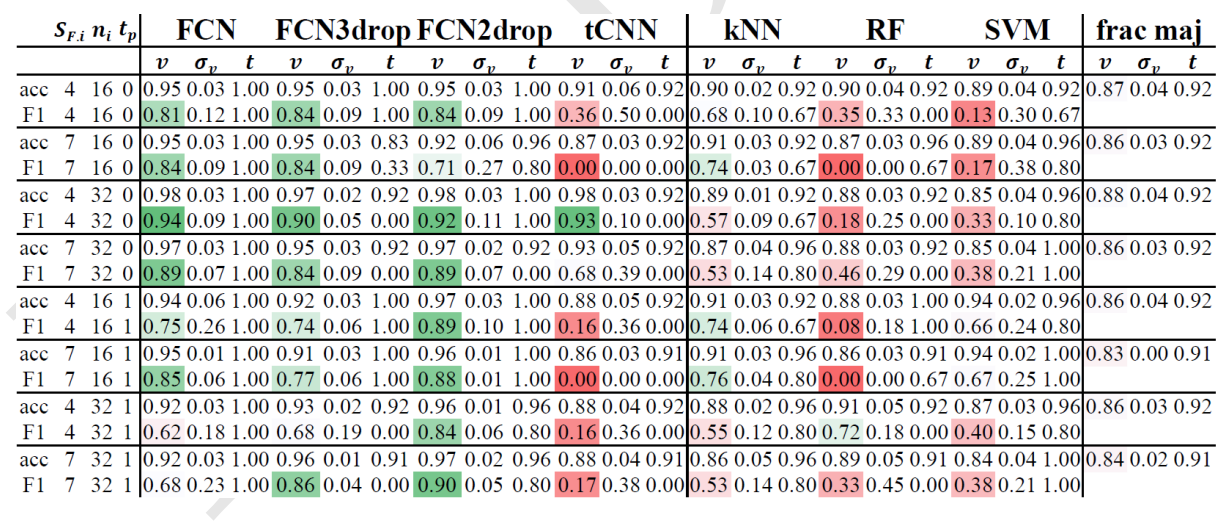

Of the 11520 trained models, we present the results for $\delta t=30 \mathrm{~min}, n_{i}=$ $[16,32], t_{p}=[0,1]$, and $n_{o}=4$ when predicting fault code $\mathbf{S}_{F_{4}}$ (malfunction of a power converter controller) and $\mathbf{S}_{F_{7}}$ (failure of a current measurement device), in Table 2 which showed high accuracy for both an on-line $\left(t_{p}=1\right)$ and postmortem $\left(t_{p}=0\right)$ use.

The FCN networks show F1 close to 1. Note that the models were trained with as few as 17 class ' 1 ' examples on average for both the validation folds and the final training on the whole data-set. The F1 for the tCNN networks ranges 
from zero to close to one for different problem parameters. Its performance might be improved by additional tuning of the network parameters. The F1 of classical models is mostly smaller than 0.5 which is evidence that for infrastructures with discrete data deep networks outperform traditional machine learning classifiers. Applying dropout does not significantly improve the performance.

An input relevance example is shown in Fig. 7b. The FCN filters less inputs as relevant than the SVM. Still both have comparable predictive performance. Analyzing the input relevance plots with system experts, the system behaviour could be recovered and non-trivial insights obtained. However, additional manual inspection of data was necessary to fully explore the potential of these insights.

Overall, the framework demonstrates good performance in predicting system failures. However, only a subset of failures are predictable. Most likely this is due to insufficient observability of relevant processes within the logged data, which was neither conceived nor stored with the goal of using it for failure predictions. Furthermore, in all systems there are randomly occurring errors that do not necessarily have precursors.

The quality of explanations of predictions is more difficult to assess than for the synthetic scenarios where the true failure mechanism is known. However, system experts confirmed that the input relevance provides useful insights for failure analysis, which gives confidence in the method and approach for future analyses.

\section{Summary, Conclusions and Outlook}

Our data driven framework identifies failure mechanisms in complex infrastructures, such as particle accelerators. Using multivariate time series data from infrastructure monitoring signals, a predictive model is learned with deep convolutional neural networks and classical machine learning algorithms. Explainable AI methods, such as layer wise relevance propagation, identify the most relevant failure precursors in the monitoring data. This has the potential to allow a more focused trouble-shooting of operational incidents.

The framework is applied to synthetic and real world data-sets. With synthetic data, the framework correctly isolates relevant failure precursors from up to hundred time series with as few as ten examples of failures to learn from and is able to recover interactions between multiple sub-systems. With real-world data, deep neural networks predict failures with F1 scores close to 1 for particle accelerator problems at a bare minimum of data preprocessing and problemadaptation. Non-trivial system behaviour could be identified from the explanation mechanism. Fully Convolutional Neural Networks outperform classical ML methods in our experiments. Explainable deep learning proves to be a promising tool for future fault prognostics applications in particle accelerators.

For future research, the experimental verification will be extended to further prognostics and diagnostics tasks inside and outside the particle accelerator

domain. Since the framework does not rely upon specific insights from particle accelerators, it can be assumed that it performs equally well in other fields of 
application. An integrated treatment of uncertainty quantification is currently missing, but would be valuable in limited data scenarios. To solve the limitations due to the lack of failure data, transfer learning and few-shot learning could be investigated. Continuous changes in the infrastructure causing concept drifts could be tackled by re-learning approaches.

\section{References}

1. Abdul, A., Vermeulen, J., Wang, D., Lim, B.Y., Kankanhalli, M.: Trends and trajectories for explainable, accountable and intelligible systems: An hci research agenda. In: Proceedings of the $2018 \mathrm{CHI}$ conference on human factors in computing systems. pp. 1-18 (2018)

2. Amarasinghe, K., Kenney, K., Manic, M.: Toward explainable deep neural network based anomaly detection. In: 2018 11th International Conference on Human System Interaction (HSI). pp. 311-317. IEEE (2018)

3. Apollonio, A., Cartier-Michaud, T., Felsberger, L., Müller, A., Todd, B.: Machine learning for early fault detection in accelerator systems (Jan 2020), http://cds.cern.ch/record/2706483

4. Bach, S., Binder, A., Montavon, G., Klauschen, F., Müller, K.R., Samek, W.: On pixel-wise explanations for non-linear classifier decisions by layer-wise relevance propagation. PloS one 10(7) (2015)

5. Bach-Andersen, M., Rømer-Odgaard, B., Winther, O.: Deep learning for automated drivetrain fault detection. Wind Energy 21(1), 29-41 (2018)

6. Bergmeir, C., Benítez, J.M.: On the use of cross-validation for time series predictor evaluation. Information Sciences 191, 192-213 (2012)

7. Calderini, F., Stapley, N., Tyrell, M., Pawlowski, B.: Moving towards a common alarm service for the lhc era. Tech. rep. (2003)

8. Eichler, M.: Causal inference with multiple time series: principles and problems. Philosophical Transactions of the Royal Society A: Mathematical, Physical and Engineering Sciences 371(1997), 20110613 (2013)

9. Fawaz, H.I., Forestier, G., Weber, J., Idoumghar, L., Muller, P.A.: Deep learning for time series classification: a review. Data Mining and Knowledge Discovery 33(4), 917-963 (2019)

10. Felsberger, L., Todd, B., Kranzlmüller, D.: Power converter maintenance optimization using a model-based digital reliability twin paradigm. In: 2019 4th International Conference on System Reliability and Safety (ICSRS). pp. 213-217. IEEE (2019)

11. Fronza, I., Sillitti, A., Succi, G., Terho, M., Vlasenko, J.: Failure prediction based on log files using random indexing and support vector machines. Journal of Systems and Software 86(1), 2-11 (2013)

12. Fulp, E.W., Fink, G.A., Haack, J.N.: Predicting computer system failures using support vector machines. WASL 8, 5-5 (2008)

13. Guo, J., Li, Z., Li, M.: A review on prognostics methods for engineering systems. IEEE Transactions on Reliability (2019)

14. Ioffe, S., Szegedy, C.: Batch normalization: Accelerating deep network training by reducing internal covariate shift. arXiv preprint arXiv:1502.03167 (2015)

15. Khan, S., Yairi, T.: A review on the application of deep learning in system health management. Mechanical Systems and Signal Processing 107, 241-265 (2018) 
16. Leahy, K., Hu, R.L., Konstantakopoulos, I.C., Spanos, C.J., Agogino, A.M., O'Sullivan, D.T.: Diagnosing and predicting wind turbine faults from scada data using support vector machines. International Journal of Prognostics and Health Management 9(1), 1-11 (2018)

17. Liu, C., Lore, K.G., Sarkar, S.: Data-driven root-cause analysis for distributed system anomalies. In: 2017 IEEE 56th Annual Conference on Decision and Control (CDC). pp. 5745-5750. IEEE (2017)

18. Montavon, G.: Gradient-based vs. propagation-based explanations: an axiomatic comparison. In: Explainable AI: Interpreting, Explaining and Visualizing Deep Learning, pp. 253-265. Springer (2019)

19. Mori, J., Mahalec, V., Yu, J.: Identification of probabilistic graphical network model for root-cause diagnosis in industrial processes. Computers \& chemical engineering 71, 171-209 (2014)

20. Niemi, A., Apollonio, A., Ponce, L., Todd, B., Walsh, D.J.: CERN Injector Complex Availability 2018 (Feb 2019), https://cds.cern.ch/record/2655447

21. Pedregosa, F., Varoquaux, G., Gramfort, A., Michel, V., Thirion, B., Grisel, O., Blondel, M., Prettenhofer, P., Weiss, R., Dubourg, V., Vanderplas, J., Passos, A., Cournapeau, D., Brucher, M., Perrot, M., Duchesnay, E.: Scikit-learn: Machine learning in Python. Journal of Machine Learning Research 12, 2825-2830 (2011)

22. Qiu, H., Liu, Y., Subrahmanya, N.A., Li, W.: Granger causality for time-series anomaly detection. In: 2012 IEEE 12th international conference on data mining. pp. 1074-1079. IEEE (2012)

23. Ran, Y., Zhou, X., Lin, P., Wen, Y., Deng, R.: A survey of predictive maintenance: Systems, purposes and approaches. arXiv preprint arXiv:1912.07383 (2019)

24. Saeki, M., Ogata, J., Murakawa, M., Ogawa, T.: Visual explanation of neural network based rotation machinery anomaly detection system. In: 2019 IEEE International Conference on Prognostics and Health Management (ICPHM). pp. 1-4. IEEE (2019)

25. Salfner, F., Lenk, M., Malek, M.: A survey of online failure prediction methods. ACM Computing Surveys (CSUR) 42(3), 1-42 (2010)

26. Samek, W., Binder, A., Montavon, G., Lapuschkin, S., Müller, K.R.: Evaluating the visualization of what a deep neural network has learned. IEEE transactions on neural networks and learning systems 28(11), 2660-2673 (2016)

27. Serio, L., Antonello, F., Baraldi, P., Castellano, A., Gentile, U., Zio, E.: A smart framework for the availability and reliability assessment and management of accelerators technical facilities. In: Journal of Physics: Conference Series. vol. 1067, p. 072029. IOP Publishing (2018)

28. Vilalta, R., Ma, S.: Predicting rare events in temporal domains. In: 2002 IEEE International Conference on Data Mining, 2002. Proceedings. pp. 474-481. IEEE (2002)

29. Wang, Z., Yan, W., Oates, T.: Time series classification from scratch with deep neural networks: A strong baseline. In: 2017 International joint conference on neural networks (IJCNN). pp. 1578-1585. IEEE (2017)

30. Zhao, B., Lu, H., Chen, S., Liu, J., Wu, D.: Convolutional neural networks for time series classification. Journal of Systems Engineering and Electronics 28(1), 162-169 (2017)

31. Zhao, G., Zhang, G., Ge, Q., Liu, X.: Research advances in fault diagnosis and prognostic based on deep learning. In: 2016 Prognostics and System Health Management Conference (PHM-Chengdu). pp. 1-6. IEEE (2016) 
32. Zhu, B., Wang, G., Liu, X., Hu, D., Lin, S., Ma, J.: Proactive drive failure prediction for large scale storage systems. In: 2013 IEEE 29th symposium on mass storage systems and technologies (MSST). pp. 1-5. IEEE (2013)

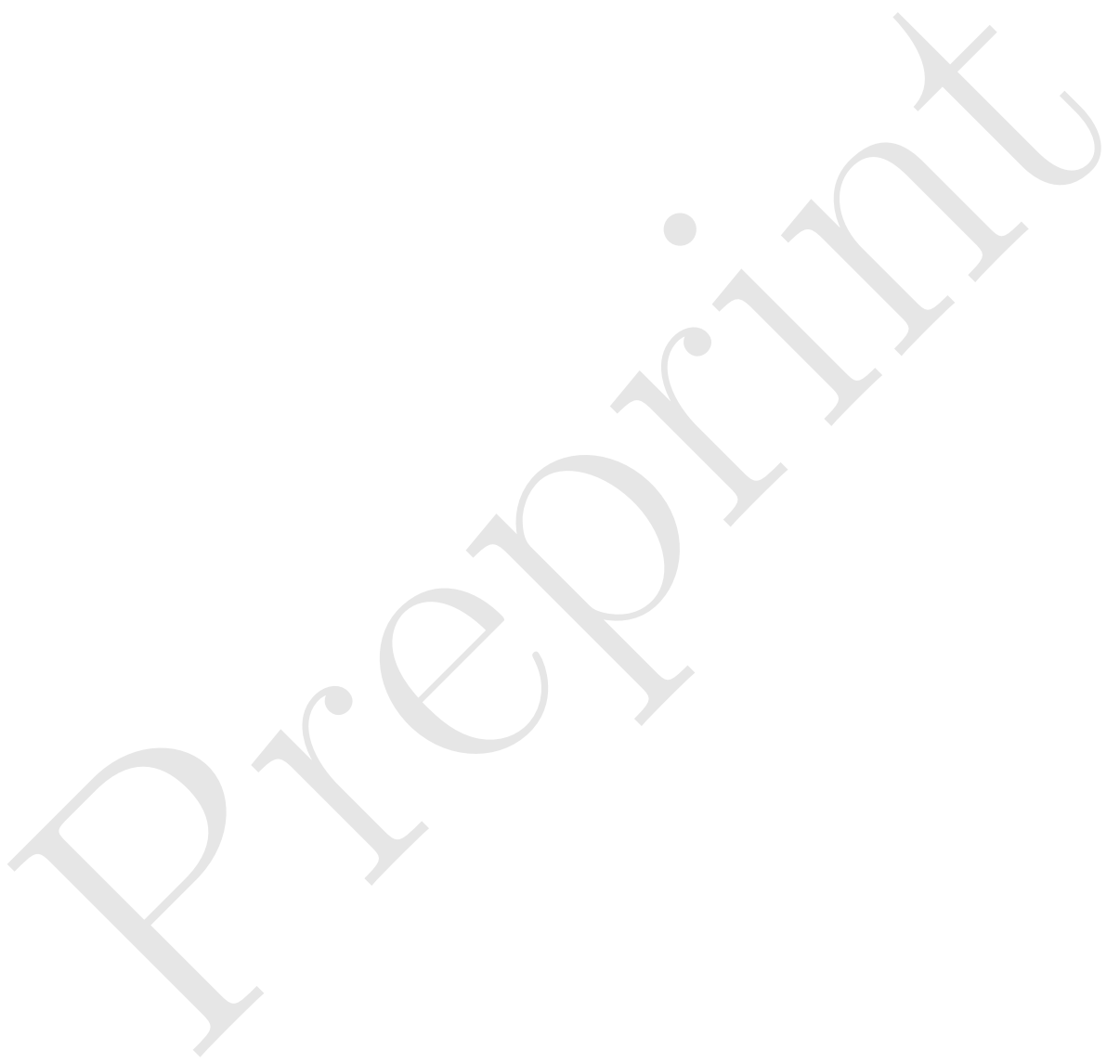

